Rel axat i on ti me br oadeni ng on emi ssi on spect rum of a Zn- doped p-type GaAs i nj ect i on I aser

\begin{tabular}{|l|l|}
\hline 著者 & $\begin{array}{l}\text { Yamada M nor u, Tanaka Aki ra, Mor i ya Ki yoshi , } \\
\text { Kado Yui chi }\end{array}$ \\
\hline $\begin{array}{l}\text { j our nal or } \\
\text { publ i cat i on ti t l e }\end{array}$ & Appl i ed Physi cs Let ter s \\
\hline vol une & 43 \\
\hline number & 9 \\
\hline page range & 818 - 20 \\
\hline year & 1983 - 11- 01 \\
\hline URL & ht t p: //hdl . handl e. net /2297/7670 \\
\hline
\end{tabular}




\title{
Relaxation time broadening on emission spectrum of a Zn-doped $p$-type GaAs injection laser
}

\author{
Minoru Yamada, Akira Tanaka, Kiyoshi Moriya, ${ }^{\text {a) }}$ and Yuichi Kado ${ }^{\text {b) }}$ \\ Department of Electrical Engineering, Faculty of Technology, Kanazawa University, Kanazawa 920, Japan
}

(Received 4 April 1983; accepted for publication 24 August 1983)

Spontaneous emission and lasing gain profiles of semiconductor injection lasers which have $\mathrm{Zn}$ doped $\mathrm{GaAs}$ active regions were experimentally determined, and the tailing phenomenon into the band gap of these profiles was theoretically explained as a result of relaxation time broadening due to scattering of electrons and holes. Especially, the tail of the gain profile was found to show a concave shape, which was better explained by the relaxation time broadening model than by the band tail state model.

PACS numbers: 42.55.Px, 78.45. + h, 78.55. - m, 78.50.Ge

Spontaneous emission and optical gain profiles of a semiconductor injection laser give information about the electron transition mechanism. So far, tailing phenomena, which are observed in spontaneous emission and optical gain profiles, have been considered due to the band tail state (BTS) broadening into the band gap. Theoretical analysis of the BTS was given by Kane, Halperin, and Lax. ${ }^{1,2}$ Analysis of lasing gain and spontaneous emission profiles were presented by Lasher, Stern, and Casey based on the BTS model. ${ }^{3-5}$

However, direct application of the BTS model to injection lasers with undoped active regions appears to be diff-

\footnotetext{
a) Presently at Nanao Electric Co. Ltd., Japan.

b) Presently at Tohoku University, Japan.
}

cult because energy fluctuations due to impurity atoms should be negligible in an undoped semiconductor crystal. The effects of measured tailing phenomena on spontaneous emission from undoped active regions have been well explained as a result of relaxation time broadening (RTB) which is characterized by scattering of electrons and holes. ${ }^{6-8}$ So far, this RTB effect has not been taken into account explicitly in the BTS analysis of the optical transition of electron. ${ }^{1-5}$

The scattering time of electrons or holes in a heavily doped $p$-type GaAs crystal is less than $4 \times 10^{-14} \mathrm{~s}$, which gives RTB into the band gap of more than $17 \mathrm{meV}$ taken at the half-width. Consequently experimentally observed emission profiles from an impurity doped active region should 


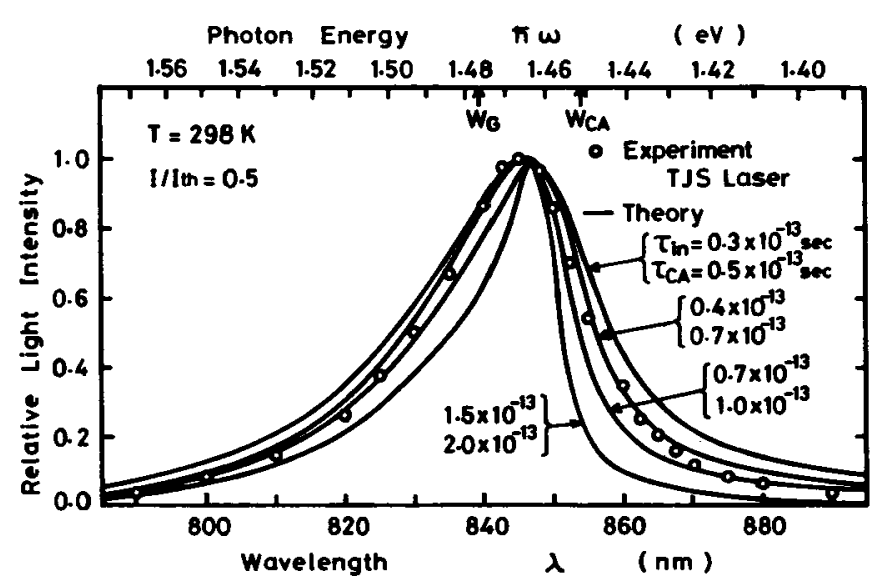

FIG. 1. Spontaneous emission profile from the laser facet. Theoretical profiles are calculated by Eqs. (2) and (3). $W_{G}$ is the band-gap energy and $W_{c A}$ is the energy difference between the conduction-band edge and the acceptor level. Parabolic state densities of the conduction and the heavy hole bands and single energy level acceptor are assumed. The theoretical profiles reveal tailing phenomena due to the phase relaxation of electrons and holes.

show the RTB effect. In this letter we measure the spontaneous emission and lasing gain profiles of transverse junction stripe (TJS) AlGaAs laser which has a Zn-doped $\left(N_{A}=5 \times 10^{18} \sim 1 \times 10^{19} \mathrm{~cm}^{-3}\right)$ active region, and compare the data with theoretical profiles based on the RTB model. ${ }^{8,10-12}$ In our model the acceptor energy level is assumed to be discrete and not extended continuously from the valence band. As a result, our theoretical gain profiles have concave shapes in the long wavelength region, which is in good agreement with experiment. Such behavior cannot be predicted by the BTS analysis.

The spontaneous emission profile was measured for a TJS laser, which oscillates at a wavelength of $844.9 \mathrm{~nm}$ and has a threshold current level of $26 \mathrm{~mA}$ at $25^{\circ} \mathrm{C}$. The emission spectrum shows resonating mode structure with any injection current level even below the threshold current level. An envelope profile connecting the peak values of the resonating modes is given by the circles in Fig. 1. The tailing phenomenon is observed in the longer wavelength region.

The gain profiles measured for another TJS sample are given in Fig. 2. This second TJS sample has almost the same configuration as the sample in Fig. 1 and has a lasing wavelength of $840.8 \mathrm{~nm}$ and a threshold current level of $25 \mathrm{~mA}$ at $20^{\circ} \mathrm{C}$. The gain profiles were determined from output light intensity at the resonating $\left(P^{+}\right)$and the nonresonating $\left(P^{-}\right)$ wavelength by the following equation ${ }^{13}$ :

$\alpha-\alpha^{\text {loss }}-\frac{1}{L} \ln \left(\frac{1}{R}\right)=\frac{1}{L} \ln \left(\frac{\left(P^{+} / P^{-}\right)^{1 / 2}+1}{\left(P^{+} / P^{-}\right)^{1 / 2}-1}\right)$,

where $\alpha$ is the gain coefficient, $\alpha^{\text {loss }}$ is the guiding loss including the free-carrier absorption and the diffraction loss, $L$ is cavity length, and $R$ is mirror reflectivity. The resolution of the monochromator was about $0.01 \mathrm{~nm}$. The measured gain profiles reveal a concave shape in the longer wavelength region. This concave shape is clearer in profiles with low injection current levels.

Theoretical calculations are also given in Figs. 1 and 2. The gain coefficient $\alpha$ is represented by a linear combination of electron transition probabilites from conduction band to heawy hole band and from conduction band to acceptor level

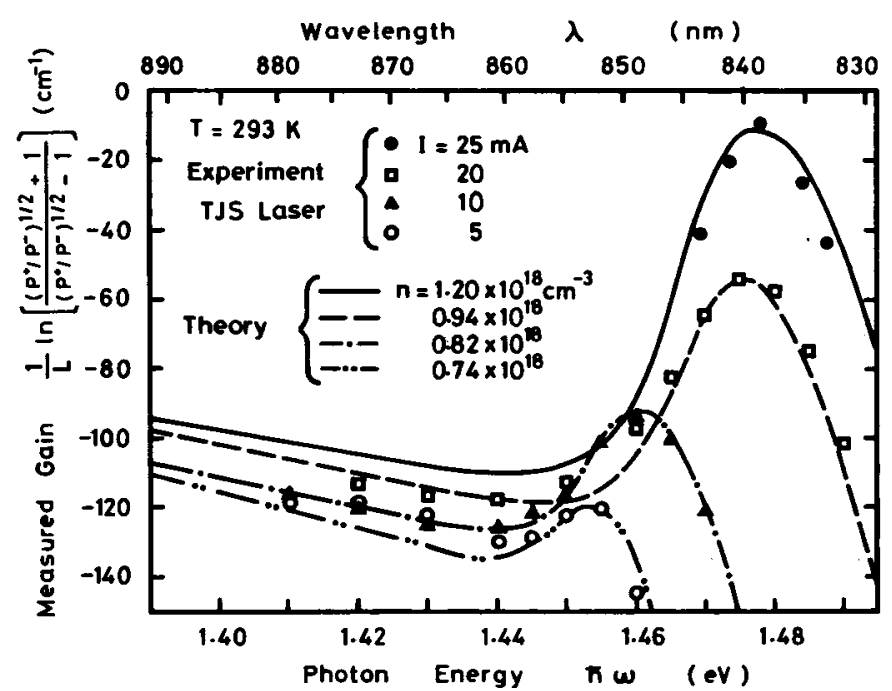

FIG. 2. Gain profiles. The experimental and theoretical profiles show concave shapes in longer wavelength regions than the gain peaks. Threshold current level of the sample is $I_{\mathrm{th}}=25 \mathrm{~mA}$. In theoretical calculations, variation of the band gap $W_{G}$ and the injected carrier density $n$ are arbitrarily chosen to fit the profiles to the experimental data.

$$
\begin{aligned}
& \text { as }^{12} \\
& \begin{aligned}
\alpha= & \int_{W_{G}}^{\infty} \frac{g_{c v}\left(W_{b}, W_{a}\right)\left[f_{c}\left(W_{b}\right)-f_{v}\left(W_{a}\right)\right]\left(\hbar / \tau_{\text {in }}\right)}{\left(W_{b a}-\hbar \omega\right)^{2}+\left(\hbar / \tau_{\text {in }}\right)^{2}} d W_{b a} \\
& +B \int_{W_{c A}}^{\infty} \frac{g_{c}\left(W_{b}\right)\left[f_{c}\left(W_{b}\right)-f_{A}\left(W_{A}\right)\right]\left(\hbar / \tau_{c A}\right)}{\left(W_{b A}-\hbar \omega\right)^{2}+\left(\hbar / \tau_{c A}\right)^{2}} d W_{b A},
\end{aligned}
\end{aligned}
$$

where $g_{c v}$ is a modified state density function to give parabolic state densities of conduction and heavy hole bands in a single equation. ${ }^{10} f_{c}$ and $f_{v}$ are Fermi-Dirac distribution functions for electrons in conduction and heavy hole bands, respectively. These two Fermi-Dirac functions are defined at injected states characterized by two quasi-Fermi levels, respectively. $W_{b a}$ is the energy difference between an energy level $W_{b}$ in the conduction band and another energy level $W_{a}$ in the heavy hole band. $\tau_{\text {in }}$ is the phase relaxation time of the electron-hole dipole and is determined by collision times of the electrons in the conduction band and holes in the heavy hole band. ${ }^{10,12}$ The electron transition between the two bands is characterized by the electron distribution function $g_{c v}\left(f_{c}-f_{v}\right)$ and by the RTB given by a Lorentz function $\left(\hbar / \tau_{\text {in }}\right) /\left[\left(\mathbf{W}_{\mathrm{ba}}-\hbar \omega\right)^{2}+\left(\hbar / \tau_{\text {in }}\right)^{2}\right]$. The second term in Eq. (2) indicates electron transition between the conduction band and the acceptor level : $\tau_{c A}$ is the relaxation time of the dipole corresponding to the latter transition mechanism. $g_{c}$ is the parabolic state density of the conduction band. $f_{A}$ is the Fermi-Dirac distribution function at the acceptor level which is assumed to be a single energy level $W_{A}$, and the electron distribution is characterized same quasi-Fermi as for the valence band. $W_{G}$ is the band gap and $W_{c A}$ is the energy difference between the conduction band and the acceptor level. Coefficients $A$ and $B$ include the dipole moment and the field confinement ratio of the active region. The acceptor density $N_{A}$ is included in $B$.

The spontaneously emitted optical field experiences lasing gain $\alpha$ and guiding loss $\alpha^{\text {loss }}$ in the active region. Thus, the light output $Q$ in case of a very low injection state is given 


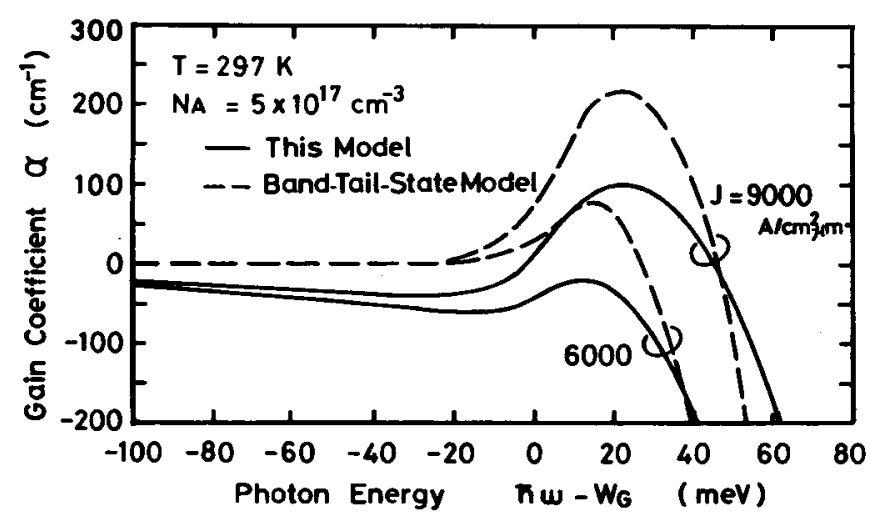

FIG. 3. Comparison of our theoretical gain profiles based on the RTB model to the profiles based on the BTS model by Stern in Ref. 4 . The profiles by the BTS model do not show the concave shape which is observed experimentally. In this analysis, $\tau_{\text {in }}=7 \times 10^{-14} \mathrm{~s}, \tau_{c A}=1 \times 10^{-13} \mathrm{~s}$, and $B /$ $A=0.11$ are assumed with $N_{A}=5 \times 10^{17} \mathrm{~cm}^{-3}$.

by $^{12}$

$$
\begin{gathered}
Q \propto\left(\alpha^{\text {loss }}-\alpha\right)^{-1}\left(A \int_{W_{G}}^{\infty} \frac{g_{c v} f_{c}\left(1-f_{v}\right)\left(\hbar / \tau_{\text {in }}\right)}{\left(W_{b a}-\hbar\right)^{2}+\left(\hbar / \tau_{\text {in }}\right)^{2}} d W_{b a}\right. \\
\left.+B \int_{W_{c A}}^{\infty} \frac{g_{c} f_{c}\left(1-f_{A}\right)\left(\hbar / \tau_{c A}\right)}{\left(W_{b A}-\hbar \omega\right)^{2}+\left(\hbar / \tau_{c A}\right)^{2}} d W_{b A}\right) .
\end{gathered}
$$

The spontaneous emission is expressed in terms of $g_{c v} f_{c}\left(1-f_{v}\right)$ and $g_{c} f_{c}\left(1-f_{A}\right)$.

Numerical examples of the spontaneous emission and the lasing gain are shown in Figs. 1 and 2 using Eqs. (3) and (2). The value of $A$ was determined to $A=3.64 \times 10^{-40} \mathrm{Jm}^{2}$ from measurements on another sample with undoped active region. " The ratio of $B$ to $A$ is determined to $B / A=1.4$ by best fitting of the experimental data. The acceptor concentration was $N_{A}=7 \times 10^{18} \mathrm{~cm}^{-3}$. In Fig. 1 the guiding loss $\alpha^{\text {loss }}$ of this sample is assumed to be $50 \mathrm{~cm}^{-1}$ and the injection current level $I$ is determined from the ratio of the injected carrier density $n$. In this analysis, no energy state lower than the band gap was assumed except for the single acceptor level. However, Fig. 1 shows a continuous tail into the band gap due to the relaxation phenomena. The relaxation times can be determined through mobility measurements and other theoretical considerations about scattering mechanisms and are estimated to be $\tau_{\text {in }}=4 \times 10^{-14} \mathrm{~s}$ and $\tau_{c A}=7 \times 10^{-14} \mathrm{~s}$ for $N_{A}=7 \times 10^{18} \mathrm{~cm}^{-3} \cdot{ }^{12,14}$ The relaxation times in this case are slightly shorter than for undoped samples ${ }^{8}$ because of electron and hole versus impurity atom scattering mechanisms. The theoretical emission profile coincides well with the experimental profile using these relaxation times.

For the theoretical calculations in Fig. 2, the values of the band gap $W_{G}$ and the injected carrier density $n$ were arbitrarily chosen for each injection level to fit the theoretical profiles to the experimental data, but the energy-level difference between the acceptor level and the valence band was assumed to be constant $\left(W_{G}-W_{c A}=24 \mathrm{meV}\right)$. The theoretical values of lasing gain in Fig. 2 are corrected by $\alpha^{\text {loss }}+(1 / L) \ln (l / R)=60 \mathrm{~cm}^{-1}$. The assumed loss value $\alpha^{\text {loss }}$ in Fig. 2 seems to be smaller than that in Fig. 1. This difference may be caused by errors in determining the true injection levels because our theory does not take into account carrier leakage phenomena or nonradiative recombination of electrons.

Our theoretical gain profiles show a concave shape, which has not been predicted by the BTS model as in Fig. 3. ${ }^{3-5}$ This concave shape indicates that the RTB is wider than the width of the population inversion characterized by the positive portion of $g_{c v}\left(f_{c}-f_{v}\right)$, then the gain profile becomes negative in the band gap due to strong absorption at higher energy levels where $g_{c v}\left(f_{c}-f_{v}\right)<0$.

Such concave shape is not always observed in all types of injection lasers. ${ }^{15}$ The shape of the carrier distribution $g_{c v}\left(f_{c}-f_{v}\right)$ is determined by injected carrier density, location of the quasi-Fermi levels, the amount of the effective masses, and the band gap. In case of AlGaAs lasers, such concave shape is observed less clearly in lasers with undoped active regions than in lasers with $p$-type active regions. ${ }^{10-12,15}$

The peak gain given by this RTB model is smaller than that by the BTS model as shown in Fig. 3. However, we cannot determine by experiments which model gives more accurate results on the values of the peak gain, because we do not know details of the carrier leakage phenomena, the nonradiative recombination or the field confinement ratio of the active region in lasers.

In this analysis, we have neglected the broadening due to the impurity levels for simplicity. The actual impurity levels should result in broadening to some extent due to overlapping of wave functions of impurity atoms and exciton interactions. For a more accurate calculation of emission and absorption profiles, the broadened energy states and higher order collisions of carriers should be taken into account. However, in this letter, we have demonstrated that the measured spontaneous emission and gain profiles are explained better by the RTB model than the BTS model.

The authors thank Professor Y. Suematsu of Tokyo Institute of Technology, Professor M. Suzuki of Kanazawa University, and Dr. R. F. Leheny of Bell Laboratories for their helpful advices and discussions, and Dr. W. Suzaki, D. K. Ikeda, and Dr. S. Takamiya of Mitubishi Electric for support of the laser samples.

'E. O. Kane, Phys. Rev. 131, 79 (1963).

${ }^{2}$ B. I. Halperin and M. Lax, Phys. Rev. 148, 722 (1966)

${ }^{3}$ G. Lasher and F. Stern, Phys. Rev. A 133, 553 (1964).

${ }^{4}$ F. Stern, J. Appl. Phys. 47, 5382 (1976).

${ }^{5}$ H. C. Casey, Jr., and F. Stern, J. Appl. Phys. 47, 631 (1976).

${ }^{6}$ P. T. Lansberg, Phys. Status Solidi 15, 623 (1966).

${ }^{7}$ H. Nakashima, N. Chinone, and R. Ito, J. Appl. Phys. 46, 3092 (1975).

${ }^{8}$ M. Yamada, H. Ishiguro, and H. Nagato, Jpn. J. Appl. Phys. 19, 135 (1980).

${ }^{9}$ H. Namizaki, H. Kan, M. Ishii, and A. Ito, J. Appl. Phys. 45, 2785 (1974).

${ }^{10} \mathrm{M}$. Yamada and Y. Suematsu, J. Appl. Phys. 52, 2653 (1981).

"M. Yamada and H. Ishiguro, Jpn. J. Appl. Phys. 20, 1279 (1981).

${ }^{12}$ M. Yamada, R. Kawachi, A. Tanaka, H. Sasaki, and H. Ishiguro (unpublished).

${ }^{13}$ B. Hakki and T. L. Paoli, J. Appl. Phys. 44, 4113 (1973).

${ }^{14} \mathrm{M}$. Yamanishi, Technical Report from Institute of Electron. Commun. Eng. Japan OQE80-103 (1980) (Japanese).

${ }^{15}$ C. H. Henry, R. A. Logan, and F. R. Meritt, J. Appl. Phys. 51, 3042 (1980). 